\title{
Discrete Helmholtz Decomposition for Electric Current Volume Integral Equation Formulation
}

\section{Markkanen, Johannes}

2014

Markkanen , J 2014 , ' Discrete Helmholtz Decomposition for Electric Current Volume Integral Equation Formulation ' , IEEE Transactions on Antennas and Propagation , vol. 62 , no. 12 , pp. 6282-6289 . https://doi.org/10.1109/TAP.2014.2364614

http://hdl.handle.net/10138/152742

https://doi.org/10.1109/TAP.2014.2364614

acceptedVersion

Downloaded from Helda, University of Helsinki institutional repository.

This is an electronic reprint of the original article.

This reprint may differ from the original in pagination and typographic detail.

Please cite the original version. 


\title{
Discrete Helmholtz Decomposition for Electric Current Volume Integral Equation Formulation
}

\author{
Johannes Markkanen
}

\begin{abstract}
A volume integral equation formulation for the equivalent current is investigated by decomposing the $L_{2}$ conforming unknown current into orthogonal functions. The decomposition shows that the solenoidal, irrotational and harmonic subspaces scale differently with respect to the material parameter. This has a negative effect on the conditioning of the system, and thus, the convergence of the iterative solution slows down with increasing permittivity. We construct discrete decomposition operators, and use them as a preconditioner for the electric current volume integral equation. The eigenvalues of the resulting system are almost independent on the permittivity. Numerical examples show that the proposed preconditioner improves the condition number and decreases the number of iterations required to solve the system. However, efficient evaluations of the projection operators require additional regularization techniques such as abgebraic multigrid preconditioners.
\end{abstract}

Index Terms-Basis functions, method of moments, preconditioning, volume integral equations.

\section{INTRODUCTION}

$\mathbf{E}$ LECTROMAGNETIC scattering problems involving high-contrast inhomogeneous dielectric materials may arise from many different fields in science and engineering such as bioelectromagnetics, remote sensing, metamaterials, communication, etc. Mathematically, scattering problems can be formulated as boundary value problems for Maxwell's equations whose solution can be found through various numerical procedures, for instance the finite-element (FEM), the finite-difference time-domain (FDTD), or the surface/volume integral-equation (SIE/VIE) methods. In general, the integral equation based numerical solvers are more attractive for openregion scattering problems since the radiation condition is automatically satisfied, and therefore, the unknowns are local and restricted inside the scatterer. In the differential equation methods, the unknowns are global meaning that the space around the objects needs to be modelled and the region of interest must be terminated by a proper boundary condition.

VIE methods are commonly used for solving scattering problems of general inhomogeneous media since material inhomogeneities can be treated trivially. SIE methods are applicable for piecewise homogeneous materials but they might become too complicated when the number of different material domains increases since each sub domain requires the different

Manuscript received yyy; revised zzz.

The research has been funded by the ERC Advanced Grant No 320773 entitled "Scattering and Absorption of Electromagnetic Waves in Particulate Media" (SAEMPL), and the Academy of Finland No. 13259950 entitled "Computational and mathematical models for electromagnetic wave interaction with complex materials".

The author is with the Department of Physics, University of Helsinki e-mail: johannes.markkanen@helsinki.fi
Green's function. In the VIEs, only the Green's function of the background is needed, and that simplifies the analysis.

To solve the VIEs, the method of moments (MoM) is usually applied. In the MoM, the original integral equation is converted into a discrete system of linear equations with $N$ equations and unknowns. A direct solution of such a system would require $\mathcal{O}\left(N^{3}\right)$ operations and $\mathcal{O}\left(N^{2}\right)$ memory, and therefore, computationally it is too demanding to be used for any practical applications. Fortunately, due to the rapid development of sophisticated fast methods such as the multilevel fast multipole algorithm (MLFMA), and the FFTbased adaptive integral method (AIM) or the pre-corrected FFT method (pFFT) [1]-[5], VIEs have become very attractive choice for modeling inhomogeneous dielectric materials. In these techniques, a huge system of linear equations is solved iteratively, and the matrix-vector multiplication required in each iteration step is accelerated by the abovementioned algorithms. The computational complexity of all these algorithms scale roughly as $\mathcal{O}(N \log N)$ per iteration, and the memory consumption as $\mathcal{O}(N)-\mathcal{O}(N \log N)$. Hence, to obtain an efficient solver, the number of iterations required to solve the system should be minimized, and a poor convergence rate can be a major bottleneck of these algorithms. Especially, it is important that the number of iterations is independent on the mesh density.

It is well known that when the VIEs are discretized with the standard techniques, the iterative solvers tend to converge very slowly or even stall their convergence as the permittivity of the object increases [6]. The main reason for this is that the spectral radius of the volume integral operator grows with increasing permittivity [7]-[12]. Intuitively, it can be understood as different scaling of the solenoidal, irrotational and harmonic parts of the source current with respect to the permittivity. In addition, the spectrum of the discretized integral operator might not be the same as the spectrum of the original integral operator. The spectrum of the discrete operator also depends on the choice of the basis and testing functions, and the employed inner product [13].

In this paper, we review the spectral properties of the volume integral operator by applying an orthogonal decomposition, i.e., the Helmholtz decomposition. The Helmholtz decomposition shows that the solenoidal, irrotational, and harmonic subspaces scale differently with respect to the permittivity. Consequently, the condition of the system matrix is also affected by the permittivity function. This explains a poor convergence of the iterative solvers when the permittivity is large in amplitude. Thus, we propose a preconditioner that is based on a discrete Helmholtz decomposition. The discrete decomposition is obtained by constructing projection operators 
that derive from the standard solenoidal and irrotational basis functions. This technique leads to a well-posed system matrix whose condition number is independent on the mesh density and almost independent on the permittivity, which is verified by various numerical examples. In addition, we discuss how to compute the projection operators efficiently, and it is shown that additional regularization techniques, such as algebraic multigrids, are required.

\section{FORMULATIONS}

Consider a time-harmonic $(\exp (-i \omega t))$ electromagnetic wave scattering by a linear inhomogeneous three-dimensional object bounded by domain $\Omega$ in a background medium with constants $\epsilon_{0}$ and $\mu_{0}$. The total time-harmonic electric field $\boldsymbol{E}$ can be expressed via the volume equivalence principle as [5]

$$
\boldsymbol{E}=\boldsymbol{E}^{i n c}+\frac{-1}{i \omega \epsilon_{0}}\left(\nabla \nabla+k_{0}^{2} \overline{\bar{I}}\right) \cdot \mathcal{V}(\boldsymbol{J}),
$$

where $\boldsymbol{E}^{i n c}$ is the incident electric field, and $k_{0}=\omega \sqrt{\epsilon_{0} \mu_{0}}$ is the wavenumber in the background medium. The equivalent electric polarization current is defined as

$$
\boldsymbol{J}(\boldsymbol{r})=-i \omega \varepsilon_{0}\left(\epsilon_{r}(\boldsymbol{r})-1\right) \boldsymbol{E}(\boldsymbol{r}),
$$

where $\epsilon_{r}(\boldsymbol{r})$ is the relative permittivity function. The volume potential operator in (1) can be expressed as

$$
\mathcal{V}(\boldsymbol{F})(\boldsymbol{r})=\int_{\Omega} G_{0}\left(\boldsymbol{r}, \boldsymbol{r}^{\prime}\right) \boldsymbol{F}\left(\boldsymbol{r}^{\prime}\right) d V^{\prime},
$$

where $G_{0}$ is the Green's function of the background.

From representation (1) and the definition of the equivalent current (2), the volume integral equation formulation for the electric current (JVIE) can be derived:

$$
\boldsymbol{J}^{i n c}=\boldsymbol{J}-\left(\epsilon_{r}-1\right)\left(\nabla \nabla+k_{0}^{2} \overline{\bar{I}}\right) \cdot \mathcal{V}(\boldsymbol{J}) .
$$

Due to the finite energy assumption, the equivalent current $J$ must belong to the space $L^{2}(\Omega)^{3}$ [14], i.e., the space of square integrable vector functions in $\Omega$. The above equation (4) is well-posed from $L^{2}(\Omega)^{3}$ to $L^{2}(\Omega)^{3}$ assuming the permittivity function is realistic [11].

\section{ORTHOGONAL DECOMPOSITION}

Any vector field $\boldsymbol{f} \in L^{2}(\Omega)^{3}$ can be decomposed into the irrotational, solenoidal and harmonic parts as [10]

$$
\boldsymbol{f}=\nabla p+\nabla \times \boldsymbol{w}+\nabla h,
$$

where $p \in H_{0}^{1}(\Omega), \boldsymbol{w} \in H_{0}^{\text {curl }}(\Omega)^{3}$, and $h \in H^{\text {harm }}(\Omega)$ with

$$
\begin{gathered}
H_{0}^{1}(\Omega)=\left\{p \in L^{2}(\Omega), \nabla p \in L^{2}(\Omega)^{3},\left.p\right|_{\partial \Omega}=0\right\}, \\
H_{0}^{\text {curl }}(\Omega)^{3}=\left\{\boldsymbol{w} \in L^{2}(\Omega)^{3}, \nabla \times \boldsymbol{w} \in L^{2}(\Omega)^{3}, \boldsymbol{n} \times\left.\boldsymbol{w}\right|_{\partial \Omega}=0\right\}, \\
H^{\text {harm }}(\Omega)=\left\{h \in H^{1}(\Omega), \nabla^{2} h=0\right\}, \\
H^{1}(\Omega)=\left\{g \in L^{2}(\Omega), \nabla g \in L^{2}(\Omega)^{3}\right\},
\end{gathered}
$$

and $L^{2}(\Omega)$ is the space of square integrable functions in $\Omega$. It is worth noting that gradients of harmonics correspond boundary values and can be represented as $\nabla H^{\text {harm }}=\nabla H^{1} \cap \nabla \times H^{\text {curl }}$ where the spaces $H^{1}$ and $H^{c u r l}$ are defined in (6) and (7) but without vanishing boundary values [15].

Properties of the electric current volume integral equation formulation (4) is studied by decomposing the unknown current $\boldsymbol{J}$ as

$$
\boldsymbol{J}=\boldsymbol{J}^{\text {sol }}+\boldsymbol{J}^{\text {irr }}+\boldsymbol{J}^{\text {harm }},
$$

with $\boldsymbol{J}^{\text {sol }}=\nabla \times \boldsymbol{w}, \boldsymbol{J}^{\text {irr }}=\nabla p$, and $\boldsymbol{J}^{\text {harm }}=\nabla h$. For the solenoidal part $\left(\boldsymbol{J}^{\text {sol }}\right)$, the equation (4) reduces to

$$
\boldsymbol{J}^{i n c}=\boldsymbol{J}^{s o l}-\left(\epsilon_{r}-1\right) k_{0}^{2} \mathcal{V}\left(\boldsymbol{J}^{s o l}\right),
$$

since by integrating by parts

$$
\nabla \nabla \cdot \mathcal{V}\left(\boldsymbol{J}^{s o l}\right)=\nabla \int_{\Omega} G_{0} \nabla \cdot \boldsymbol{J}^{s o l} \mathrm{~d} V-\int_{\partial \Omega} G_{0} \boldsymbol{n} \cdot \boldsymbol{J}^{s o l} \mathrm{~d} S,
$$

and from the definition of $\boldsymbol{J}^{\text {sol }}$, we can see that $\nabla \cdot \boldsymbol{J}^{\text {sol }}=0$ and $\boldsymbol{n} \cdot \boldsymbol{J}^{\text {sol }}=0$, on $\partial \Omega$, hence $\nabla \nabla \cdot \mathcal{V}\left(\boldsymbol{J}^{s o l}\right)$ is identically zero. The operator $\mathcal{V}\left(\boldsymbol{J}^{\text {sol }}\right)$ is compact from $L^{2}(\Omega)^{3}$ to itself [11]. If $\left(\epsilon_{r}-1\right)$ is bounded, the product $\left(\epsilon_{r}-1\right) \mathcal{S}(\boldsymbol{J})$ is compact, and the equation (11) is of the form "identity + compact". This means that the essential spectrum is discrete, and the eigenvalues of the discretized operator should accumulate to one on the complex plane.

To analyze the irrotational part $\left(\boldsymbol{J}^{i r r}=\nabla p\right)$, we use the fact that [16]

$$
\left(\nabla \nabla+k_{0}^{2} \overline{\bar{I}}\right) \cdot \mathcal{V}(\boldsymbol{J})=\nabla \times \nabla \times \mathcal{V}(\boldsymbol{J})-\boldsymbol{J},
$$

to write the equation (4) as follows

$$
\boldsymbol{J}^{i n c}=\epsilon_{r} \boldsymbol{J}-\left(\epsilon_{r}-1\right) \nabla \times \nabla \times \mathcal{V}(\boldsymbol{J}) .
$$

Integrating by parts and using Stokes theorem, the double curl operator can be written as

$$
\begin{aligned}
\nabla \times \nabla \times & \mathcal{V}\left(\boldsymbol{J}^{i r r}\right)=\nabla \times \int_{\Omega} G_{0} \nabla \times \boldsymbol{J}^{i r r} \mathrm{~d} V \\
& -\nabla \times \int_{\partial \Omega} \boldsymbol{n} \times \boldsymbol{J}^{i r r} G_{0} \mathrm{~d} S .
\end{aligned}
$$

Due to the definition of $\boldsymbol{J}^{i r r}, \nabla \times \boldsymbol{J}^{i r r}=0$ and $\boldsymbol{n} \times \boldsymbol{J}^{i r r}=$ 0 , on $\partial \Omega$, the operator $\nabla \times \nabla \times \mathcal{V}\left(\boldsymbol{J}^{i r r}\right)$ is identically zero. Thus, only the permittivity and the identity operators act on the irrotational part

$$
\boldsymbol{J}^{i n c}=\epsilon_{r} \mathcal{I}\left(\boldsymbol{J}^{i r r}\right) .
$$

The essential spectrum is discrete, and the eigenvalues depend only on the permittivity function. The accumulation point of the eigenvalues is at $\epsilon_{r}$ on the complex plane.

Finally, we need to analyze the harmonic part. Substituting the gradient of the harmonic function $\boldsymbol{J}^{\text {harm }}=\nabla h$ into the equation (4), integrating by parts, and using the fact that $\nabla$. $\nabla h=0$, the equation (4) can be written as

$\boldsymbol{J}^{\text {inc }}=\boldsymbol{J}^{\text {harm }}+\left(\epsilon_{r}-1\right)\left(\nabla \mathcal{S}\left(\gamma_{n} \boldsymbol{J}^{\text {harm }}\right)-k_{0}^{2} \mathcal{V}\left(\boldsymbol{J}^{\text {harm }}\right)\right)$,

where $\gamma_{n}$ denotes the normal trace operator, and $\mathcal{S}$ is the single layer potential: 


$$
\mathcal{S}(\boldsymbol{F})(\boldsymbol{r})=\int_{\partial \Omega} G_{0}\left(\boldsymbol{r}, \boldsymbol{r}^{\prime}\right) \boldsymbol{F}\left(\boldsymbol{r}^{\prime}\right) \mathrm{d} S^{\prime} .
$$

The operator $\mathcal{V}\left(\boldsymbol{J}^{\text {harm }}\right)$ is a compact mapping from $\nabla H^{\text {harm }}(\Omega)$ to $L^{2}(\Omega)^{3}$, and the operator $\nabla \mathcal{S}\left(\gamma_{n} \boldsymbol{J}^{\text {harm }}\right)$ is bounded from $\gamma_{n} \nabla H^{\text {harm }}(\Omega)$ to $L^{2}(\Omega)^{3}$ [7].

The normal trace operator $\gamma_{n}$ defines the following isomorphisms [10]

$$
\nabla H^{\text {harm }} \ni \boldsymbol{h} \leftrightarrow \gamma_{n} \boldsymbol{h}=\left.\boldsymbol{n} \cdot \boldsymbol{h}\right|_{\partial \Omega} \in H_{*}^{-1 / 2}(\partial \Omega),
$$

where $H_{*}^{-1 / 2}(\partial \Omega)$ is $H^{-1 / 2}(\partial \Omega)$ with zero mean value. Hence on $\nabla H^{\text {harm }}$ we can write

$$
\begin{aligned}
& \gamma_{n} \boldsymbol{J}^{\text {harm }}-\gamma_{n}\left(\left(\epsilon_{r}-1\right) \nabla \mathcal{S}\left(\gamma_{n} \boldsymbol{J}^{\text {harm }}\right)\right) \\
= & \frac{1}{2}\left(\epsilon_{r}+1\right) \mathcal{I}(f)+\left(\epsilon_{r}-1\right) \int_{\partial \Omega} \partial_{n} G f \mathrm{~d} S,
\end{aligned}
$$

with $f \in H_{*}^{-1 / 2}(\partial \Omega)$, and $\partial_{n} G$ denotes the normal derivative of the Green's function [10]. The operator $\int_{\partial \Omega} \partial_{n} G f \mathrm{~d} S$ is a common operator in the surface integral equations [8], and it is compact in $H_{*}^{-1 / 2}$ if $\partial \Omega$ is smooth. If the surface is not smooth, the operator is not compact but it is bounded [9], [10].

The essential spectrum of the complete volume integral operator (when $\partial \Omega$ is smooth) is $\sigma_{e}=\left\{1, \frac{1}{2}\left(\epsilon_{r}+1\right), \epsilon_{r}\right\}$. In addition, the spectrum contains discrete eigenvalues which are related to the resonances [12]. The accumulation points of the eigenvalues due to the decomposed spaces on the complex plane are as follows:

$$
\begin{array}{cc}
\text { Solenoidal : } & 1 \\
\text { Irrotational : } & \epsilon_{r} \\
\text { Harmonic: } & \frac{1}{2}\left(\epsilon_{r}+1\right) .
\end{array}
$$

This indicates that the spectral radius is a function of material parameter, and it may have a negative effect on the stability of the numerical solution. This explains why iterative solvers tend to slow down their convergence in the case of high-contrast materials.

\section{Discretization}

Consider discretization of the volume integral equation (4). Let us divide the volume of the dielectric object $\Omega$ with linear tetrahedral elements $k$. We define piecewise constant basis $\boldsymbol{b}_{k}^{i}$ and testing $t_{k}^{i}$ functions on the tetrahedral mesh where the superindex $i$ denotes the $x-, y$-, or $z$-component. The current is approximated as a linear combination of basis functions with coefficients $a_{k}^{i}$ as

$$
\boldsymbol{J} \approx \sum_{k, i} a_{k}^{i} \boldsymbol{b}_{k}^{i}=\sum_{k}\left(a_{k}^{x} \hat{\boldsymbol{e}}_{x}+a_{k}^{y} \hat{\boldsymbol{e}}_{y}+a_{k}^{z} \hat{\boldsymbol{e}}_{z}\right) / \sqrt{V_{k}},
$$

where $\hat{\boldsymbol{e}}_{x}, \hat{\boldsymbol{e}}_{y}, \hat{\boldsymbol{e}}_{z}$ are the unit vectors, and $V_{k}$ is the volume of tetrahedron $k$. To obtain well-behaving discrete identity operator, i.e., the identity matrix, independently on the mesh density, the basis and testing functions are scaled by $1 / \sqrt{V_{k}}$.

The continuous integral equation is converted into a discrete set of equations by a projection method with the $L^{2}$-inner product defined as

$$
\langle\boldsymbol{F}, \boldsymbol{G}\rangle_{\Omega}=\int_{\Omega} \boldsymbol{F} \cdot \boldsymbol{G} \mathrm{d} r .
$$

To guarantee the convergence of the projection method, basis functions should span the domain of the operator, and testing functions the $L^{2}$-dual space of the range of the operator [14]. In this case, both the domain and $L^{2}$-dual of range are $L^{2}(\Omega)^{3}$, therefore the Galerkin's technique with piecewise constant functions works. This can be seen by applying the Helmholtz decomposition

$$
\begin{gathered}
<\nabla p+\nabla \times \mathbf{w}+\nabla h, \mathbf{J}>=-<p, \nabla \cdot \mathbf{J}> \\
-<\mathbf{w}, \nabla \times \mathbf{J}>+<h, \hat{\mathbf{n}} \cdot \mathbf{J}>,
\end{gathered}
$$

hence the divergence, curl, and boundary values are welltested. If, for example, a point matching technique is used rather than the Galerkin, there would be no guarantee that $\nabla \cdot \boldsymbol{J}$ (charge) converges.

Taking the inner product and using integration by parts, the integral operator can be discretized as

$$
\begin{gathered}
A_{m n}^{i j}=\int_{V_{m}} \boldsymbol{t}_{m}^{i} \cdot \boldsymbol{b}_{m}^{j} \mathrm{~d} V \\
+\int_{\partial V_{m}} \boldsymbol{n} \cdot\left(\tau \boldsymbol{t}_{m}^{i}\right) \cdot \int_{\partial V_{n}} G \boldsymbol{n}^{\prime} \cdot \boldsymbol{b}_{n}^{j} \mathrm{~d} S^{\prime} \mathrm{d} S \\
-\int_{V_{m}} \boldsymbol{t}_{m}^{i} \cdot \tau k_{0}^{2} \int_{V_{n}} G \boldsymbol{b}_{n}^{j} \mathrm{~d} V^{\prime} \mathrm{d} V,
\end{gathered}
$$

where $\tau=\left(\epsilon_{r}-1\right)$. The elements of the force vector read as

$$
b_{m}^{i}=\int_{V_{m}} \boldsymbol{t}_{m}^{i} \cdot \boldsymbol{J}^{i n c} \mathrm{~d} V .
$$

Fig. 1 shows the eigenvalues of the discretized integral equation (24) at low frequency with $\epsilon_{r}=10+2 i$. Clearly, the eigenvalue distribution of the discretized operator follows the theory. There are accumulation points at 1 and at $\epsilon_{r}$ due to the solenoidal and irrotational subspaces, and the harmonic subspace creates the continuous spectrum whose center is at $\left(\epsilon_{r}+1\right) / 2$ since the operator that contains the normal derivative of the Green's function in (20) is not compact but bounded on non-smooth surfaces.

\section{A. Discrete decomposition}

To perform a discrete Helmholtz decomposition, we use similar basis-free decomposition, which was introduced by Andriulli et. al in [17], [18] to cure the low frequency breakdown in the SIE method. This approach gives rise to the basis-related dense-discretization breakdown free decomposition which is essential in the volume discretization. In the VIEs, the standard discrete Helmholtz decomposition leads to the ill-posed system matrix, since the condition number of the discrete solenoidal Gram matrix depends strongly on the mesh density [19].

First, we define transformation matrices from solenoidal, irrotational and harmonic functions to piecewise constant functions. Let us denote a transformation matrix from solenoidal to piecewise constant functions by $\overline{\bar{S}}$. Columns of the matrix $\overline{\bar{S}}$ are the coefficients of the solenoidal loop functions expressed as linear combinations of the piecewise constant functions. 


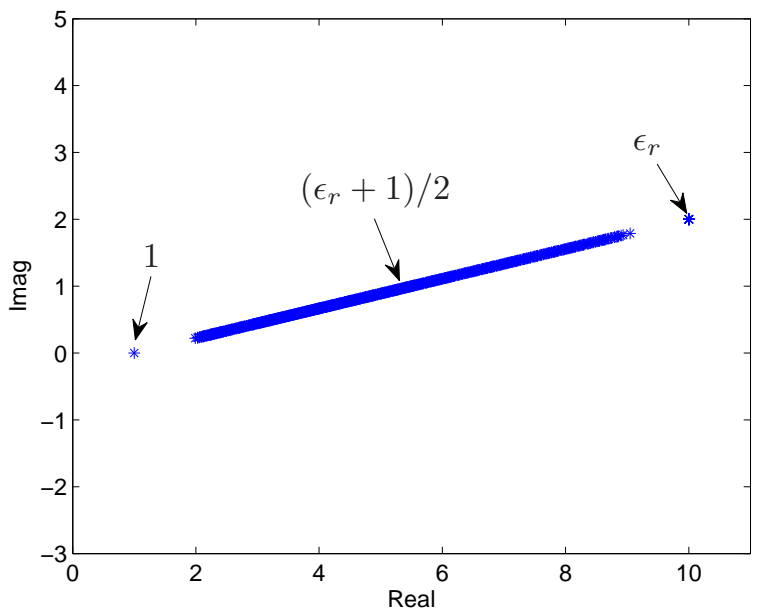

Fig. 1. The eigenvalue distribution of the discretized volume integral equation at low frequency. The spectrum contains accumulation points at 1 and at $\epsilon_{r}=10+2 i$ due to the solenoidal and irrotational subspaces as well as the continuous part (center at $\left.\left(\epsilon_{r}+1\right) / 2\right)$ due to the harmonic subspace.

The elements of the transformation matrix can be expressed as

$$
S_{k l}^{i}=<\boldsymbol{b}_{k}^{i}, \nabla \times \boldsymbol{f}_{l}>
$$

where $\boldsymbol{b}_{k}^{i}$ are piecewise constant functions defined in (21), and $f_{l}$ are the lowest order Nédélec's edge elements [20]. Since the solenoidal current lives in $\nabla \times H_{0}^{\text {curl }}(\Omega)$ the functions belonging to the boundary edges should be removed.

A transformation matrix from irrotational functions to piecewise constant functions is denoted by $\overline{\bar{I}}$ whose columns are the coefficients of the irrotational functions of the form $\nabla N_{l}$ ( $N_{l}$ are standard linear nodal functions) expressed in terms of piecewise constant functions. Elements of the matrix $\overline{\bar{I}}$ are

$$
I_{k l}^{i}=<\boldsymbol{b}_{k}^{i}, \nabla N_{l}>\text {. }
$$

The nodal functions on the boundary are set to zero due to the definition of the $\boldsymbol{J}^{i r r}$. We also note that the solenoidal and irrotational coefficient matrices are orthogonal $\overline{\bar{S}}^{T} \overline{\bar{I}}=\overline{\overline{0}}$ since the elements can be written as

$$
\begin{gathered}
\left(\overline{\bar{S}}^{T} \overline{\bar{I}}\right)_{l b}=\sum_{i, j, k, a}=<\nabla \times \boldsymbol{f}_{l}, \boldsymbol{b}_{k}^{i}><\boldsymbol{b}_{a}^{j}, \nabla N_{b}> \\
=<\nabla \times \boldsymbol{f}_{l}, \nabla N_{b}>=0,
\end{gathered}
$$

with $\boldsymbol{b}_{k}^{i} \boldsymbol{b}_{a}^{j}=1 / V_{a}$, when $a=k, i=j$, and zero otherwise.

Let us denote a transformation matrix from harmonic to piecewise constant coefficients by $\overline{\bar{H}}$. The transformation matrix $\overline{\bar{H}}$ only contains boundary elements (removed form $\overline{\bar{S}}$ and $\overline{\bar{I}}$ ), and it is orthogonal to $\overline{\bar{S}}$ and $\overline{\bar{I}}$. We do not have to compute $\overline{\bar{H}}$ since later we construct the complementary operator that finds the remaining components for gradients of harmonics.

Next, we define projection operators in the coefficient space. The coefficients of any piecewise constant function $\bar{f}$ can be represented as

$$
\bar{f}=\overline{\bar{S}} \bar{s}+\overline{\bar{I}} \bar{i}+\overline{\bar{H}} \bar{h},
$$

where $\bar{s}, \bar{i}$, and $\bar{h}$ are the coefficients of the solenoidal, irrotational, and harmonic basis functions, respectively. Due to the orthogonality of the decomposition $\overline{\bar{S}}^{T} \overline{\bar{I}}=\overline{\overline{0}}$ and $\overline{\bar{S}}^{T} \overline{\bar{H}}=\overline{\overline{0}}$, for any $\bar{f}$

$$
\overline{\bar{S}}^{T} \bar{f}=\overline{\bar{S}}^{T} \overline{\bar{S}} \bar{s}+\overline{\bar{S}}^{T} \overline{\bar{I}} \bar{i}+\overline{\bar{S}}^{T} \overline{\bar{H}} \bar{h}=\overline{\bar{S}}^{T} \overline{\bar{S}} \bar{s},
$$

is valid, and thus

$$
\bar{s}=\left(\overline{\bar{S}}^{T} \overline{\bar{S}}\right)^{+} \overline{\bar{S}}^{T} \bar{f},
$$

in which $\left(\overline{\bar{S}^{T}} \overline{\bar{S}}\right)^{+}$is the Moore-Penrose pseudoinverse of $\left(\overline{\bar{S}^{T}} \overline{\bar{S}}\right)$. The solenoidal components $\bar{f}_{s}$ of $\bar{f}$ in terms of the components of the piecewise constant functions are obtained by

$$
\bar{f}_{s}=\overline{\bar{S}} \bar{s}=\overline{\bar{S}}\left(\overline{\bar{S}}{ }^{T} \overline{\bar{S}}\right)^{+} \overline{\bar{S}}^{T} \bar{f}=\overline{\bar{P}}_{s} \bar{f}
$$

with the projection operator defined as

$$
\overline{\bar{P}}_{s}=\overline{\bar{S}}\left(\overline{\bar{S}}^{T} \overline{\bar{S}}\right)^{+} \overline{\bar{S}}^{T} \text {. }
$$

Analogously, the projection operator from the piecewise constant to the irrotational components can be written as

$$
\overline{\bar{P}}_{i}=\overline{\bar{I}}\left(\overline{\bar{I}}^{T} \overline{\bar{I}}\right)^{+} \overline{\bar{I}}^{T},
$$

and from the piecewise constant to the gradients of harmonic as

$$
\overline{\bar{P}}_{h}=\overline{\bar{H}}\left(\overline{\bar{H}}^{T} \overline{\bar{H}}\right)^{+} \overline{\bar{H}}^{T} .
$$

The tranformation matrices $\overline{\bar{S}}$ and $\overline{\bar{I}}$ are well-known matrices and easy to compute, but $\overline{\bar{H}}$ is more complicated, especially for multiply connected objects. Hence, we define a complementary projector that finds the remaining harmonic components by subtracting the solenoidal and the irrotational parts from the identity $\overline{\bar{I}}$ operator as

$$
\overline{\bar{P}}_{h}=\overline{\overline{\mathrm{I}}}-\overline{\bar{P}}_{s}-\overline{\bar{P}}_{i} .
$$

This means that the discrete functions obtained by using the projector $\overline{\bar{P}}_{h}$ are gradients of harmonics in a weak sense, i.e., they are discrete $L^{2}$ functions that are not solenoidal nor irrotational expressed as

$$
\boldsymbol{g}_{m} \in \Psi,<\nabla N_{i}, \boldsymbol{g}_{m}>=0, \text { and }<\nabla \times \boldsymbol{f}_{k}, \boldsymbol{g}_{m}>=0,
$$

in which functions $N_{i}$ and $\boldsymbol{f}_{k}$ on the boundaries are removed, and $\Psi$ is a space spanned by piecewise constant functions.

\section{B. Preconditioner}

Finally, we can construct a preconditioner for high-contrast objects by scaling the volume integral equation in the irrotational subspace by $\epsilon^{-1}$, and in the harmonic subspace by $\chi=2(\epsilon+1)^{-1}$. Inhomogeneous objects are considered as piecewise homogeneous objects, i.e., all functions in (26) and (27) related to edges and nodes laying on material interfaces as well as the outer boundary should be removed. This allows us to write the scaled equation as

$$
\begin{gathered}
\left(\overline{\bar{P}}_{s}^{T}+\overline{\bar{P}}_{i}^{T} \epsilon^{-1}+\overline{\bar{P}}_{h}^{T} \chi\right) \bar{b}= \\
\left(\overline{\bar{P}}_{s}^{T}+\overline{\bar{P}}_{i}^{T} \epsilon^{-1}+\overline{\bar{P}}_{h}^{T} \chi\right) \overline{\bar{A}}\left(\overline{\bar{P}}_{s}+\overline{\bar{P}}_{i}+\overline{\bar{P}}_{h}\right) \bar{x},
\end{gathered}
$$

where $\bar{b}, \bar{A}$, and $\bar{x}$ are the $L^{2}$-discretized incident vector (25), the system matrix (24), and the unknown coefficients, respectively. Since $\left(\overline{\bar{P}}_{s}+\overline{\bar{P}}_{i}+\overline{\bar{P}}_{h}\right)=\overline{\bar{I}}$ the equation (38) simplifies as 


$$
\left(\overline{\bar{P}}_{s}^{T}+\overline{\bar{P}}_{i}^{T} \epsilon^{-1}+\overline{\bar{P}}_{h}^{T} \chi\right) \bar{b}=\left(\overline{\bar{P}}_{s}^{T}+\overline{\bar{P}}_{i}^{T} \epsilon^{-1}+\overline{\bar{P}}_{h}^{T} \chi\right) \overline{\bar{A}} \bar{x}
$$

and using (36) we can write the system as

$$
\overline{\bar{P}}^{T} \bar{b}=\overline{\bar{P}}^{T} \overline{\bar{A}} \bar{x},
$$

where

$$
\overline{\bar{P}}=\overline{\bar{P}}_{s}(1-\chi)+\overline{\bar{P}}_{i}\left(\epsilon_{r}^{-1}-\chi\right)+\overline{\bar{I}} \chi,
$$

The eigenvalues of the above matrix are almost independent on the permittivity at low frequencies, and therefore, is more suitable for iterative solvers. In addition, the projectors used in the preconditioner lead to a stable system matrix in a sense that the eigenvalues are independent on the mesh density. The eigenvalues of the matrix $\bar{P}^{T} \overline{\bar{A}}$ at very low frequency are plotted in Fig. 2 when the permittivity is $\epsilon_{r}=10+2 i$. The spectrum for $\overline{\bar{A}}$ in the same case can be seen in Fig. 1.

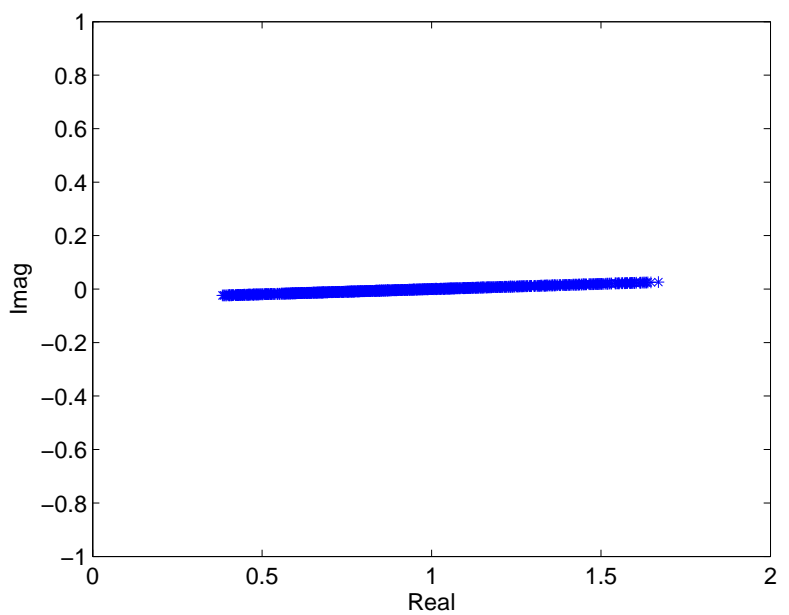

Fig. 2. The eigenvalue distribution of the discretized volume integral equation with the proposed preconditioner at low frequency. Fig. 1 shows the eigenvalues of the original operator for the same problem.

\section{Computational considerations}

In this chapter, we consider computational issues of the proposed preconditioner. For any practical application the preconditioner should be efficiently computed, i.e., complexity should be linear or almost linear for the computational time and memory requirement. Almost all practical problems require fast methods, e.g., the MLFMA or FFT-based acceleration techniques which have computational complexity of $\mathcal{O}(N \log N)$ per iteration.

The transformation matrices $\overline{\bar{S}}$ and $\overline{\bar{I}}$ are highly sparse, and should be stored in the memory. To build the projection operators, pseudo-inverses of $\overline{\bar{S}}^{T} \overline{\bar{S}}$ and $\overline{\bar{I}}^{T} \overline{\bar{I}}$ should be computed iteratively. Unfortunately, condition numbers of $\overline{\bar{S}}^{T} \overline{\bar{S}}$ and $\overline{\bar{I}}{ }^{T} \overline{\bar{I}}$ matrices depend on the mesh density, and the iterative solutions do not converge quickly when the mesh contains small and large or badly-shaped elements.

As an example Fig. 3 shows computational times for the FFT-accelerated matrix-vector multiplication $y=A x$ (one iteration step in FFT-JVIE solver), projections $\overline{\bar{P}}_{s} y$ and $\overline{\bar{P}}_{i} y$

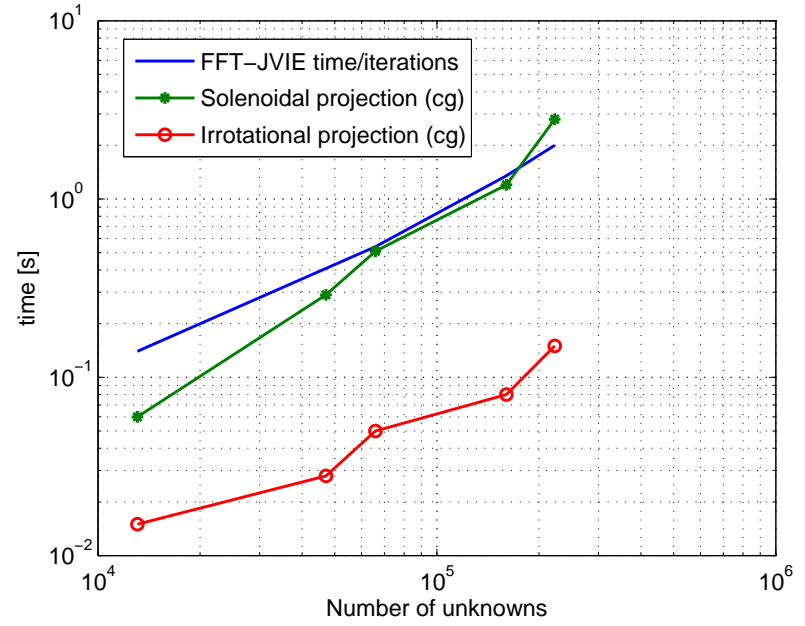

Fig. 3. Wall-times for GMRES iterations in the FFT-accelerated JVIE algorithm, and for the solenoidal and irrotational projection steps required in the preconditioner. The pseudo-inverses are computed iteratively with the CG-solver.

when the pseudo-inverses $\overline{\bar{S}}^{T} \overline{\bar{S}}$ and $\overline{\bar{I}}^{T} \overline{\bar{I}}$ are computed by the conjugate-gradient $(\mathrm{CG})$ algorithm $\left(\right.$ tol $\left.=10^{-6}\right)$. Computational times of the pseudo-inverses do not scale when a simple iterative solver is used. The number of iterations increases with the number of unknowns. Hence, preconditioners for $\overline{\bar{S}}^{T} \overline{\bar{S}}$ and $\overline{\bar{I}}^{T} \overline{\bar{I}}$ are needed.

Elements of the matrix $\overline{\bar{I}}^{T} \overline{\bar{I}}$ can be represented as

$$
\left(\overline{\bar{I}}^{T} \overline{\bar{I}}\right)_{i j}=\int_{V} \nabla N_{i} \cdot \nabla N_{j} \mathrm{~d} V,
$$

where $N_{i}$ are linear nodal basis functions. The above matrix is a well-known matrix arising from the finite-element discretization of the Poisson equation with Dirichlet boundary condition, and can be preconditioned with the standard algebraic multigrid (AMG) method [21]. In what follows, the inversion can be done nearly linearly, and the total inversion time is almost negligible.

The solenoidal projection $\overline{\bar{P}}_{s}$ requires inverting the matrix $\overline{\bar{S}}^{T} \overline{\bar{S}}$ with elements described as

$$
\left(\overline{\bar{S}}^{T} \overline{\bar{S}}\right)_{i j}=\int_{V} \nabla \times \boldsymbol{f}_{i} \cdot \nabla \times \boldsymbol{f}_{j} \mathrm{~d} V
$$

where $\boldsymbol{f}_{i}$ are the lowest order Nédélec's edge elements. This matrix appears in the magnetostatic problems when discretized with the finite-elements. The standard multigrid approach does not work in this case due to the large null-space of the curl operator. However, an efficient preconditioner can be obtained by so-called auxiliary space AMG [22], [23].

Implementing AMG preconditioners for the projection operators, one may expect significant improvements for the solution times compared with the conjugate gradient solver. In particular, the solution time should scale nearly linearly with respect to the number of unknowns. Implementing the AMG preconditioners, however, is out of scope of this paper.

\section{NUMERICAL EXAMPLES}

In this section, we study numerically how well the proposed preconditioner works in the case of high contrast dielectric 
materials. All results are calculated by the method develop in this paper, and compared with the standard J-formulation discretized with piecewise constant functions [24]. The main purpose of these examples is to demonstrate that the idea behind the preconditioner works, but we do not consider total computational times since the projectors are not efficiently computed in our implementation.

First, we investigate a small scatterer in which case the spectrum consist purely of the essential part. Consider a dielectric cone of size $r=1 \mathrm{~m}$ and height $h=2 \mathrm{~m}$ at the frequency $f=1 \mathrm{~Hz}$ (See Fig. 4). Hence, the cone is significantly smaller than the wavelength. The cone is chosen as an example because it is not a smooth object and contains elements of different sizes. Especially on top, the elements are very small compared to the ones on the bottom. The volume ratio between the largest and the smallest tetrahedron is $5.5 \cdot 10^{7}$

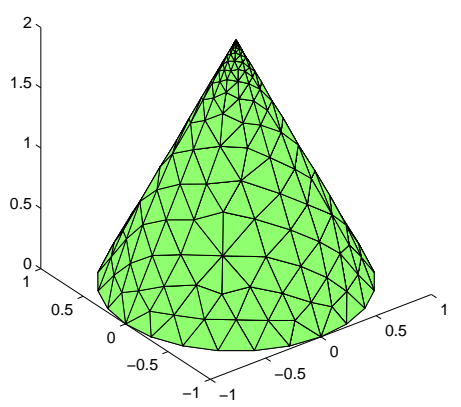

Fig. 4. Discretized cone with tetrahedral elements. The volume ratio between the largest and the smallest tetrahedron is $5.5 \cdot 10^{7}$.

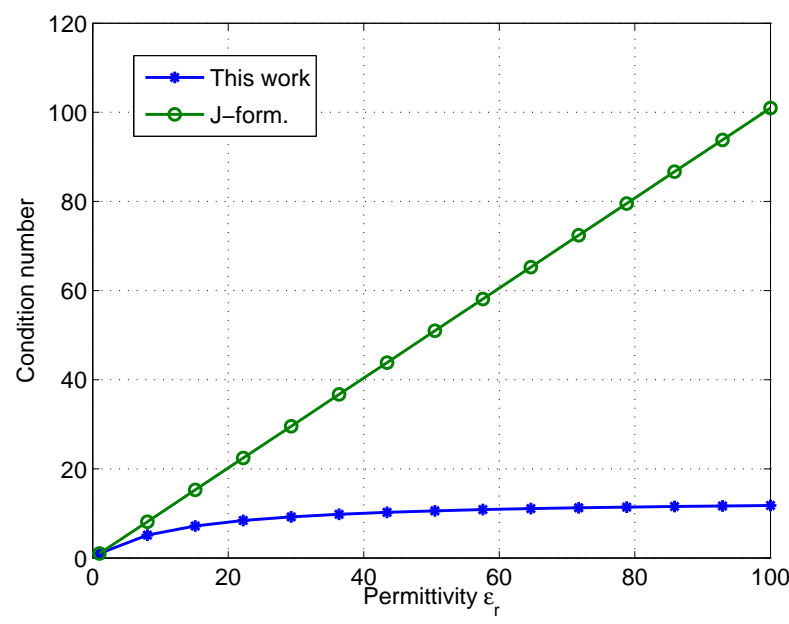

Fig. 5. Condition numbers for different permittivity values. The scatterer is a dielectric cone plotted in Fig. 4, and the frequency is $1 \mathrm{~Hz}$.

Fig. 5 shows the condition numbers of the discretized matrices with respect to the permittivity arising from the standard discretization of the J-formulation and the preconditioned one.
Clearly, the condition number of the standard J-formulation increases linearly with the permittivity. This is because the spectral radius of the operator increases linearly as a function of permittivity as discussed in Section III. By scaling the irrotational and harmonic parts, the spreading of the spectral radius can be prevented, and the condition number seems to be bounded. It is also worth noting that the size distribution of the elements do not affect the condition number at all neither in the standard discretization of the J-formulation nor the preconditioned one. This is the main reason why we have used the projection operators rather than the standard discrete Helmholtz decomposition in which case the condition number would depend on the underlying mesh.

Let us next consider a larger dielectric sphere. The size parameter of the sphere is $k_{0} a=0.5$, and the sphere is meshed with 2696 linear tetrahedra. The average element size is $h=0.2 \mathrm{~m}$ or in freespace wavelengths $h \approx 0.016 \lambda_{0}$. However, the effective wavelength in material is shorter $\left(\lambda_{e}=\lambda_{0} / \sqrt{\epsilon_{r}}\right)$, and at $\epsilon_{r}=60$, the element size is about $h \approx 0.12 \lambda_{e}$. The condition numbers for different permittivities are plotted in Fig. 6. Since the size of the object is around wavelength, the spectrum contains both the essential and discrete parts. When the permittivity is around $\epsilon_{r}=40$, the condition number peaks. This is because one discrete eigenvalue is located close to zero on the complex plane. This eigenvalue is related to the resonance solution, i.e., the diameter of the sphere equals the effective wavelength in the material $\lambda_{e}=2 a$.

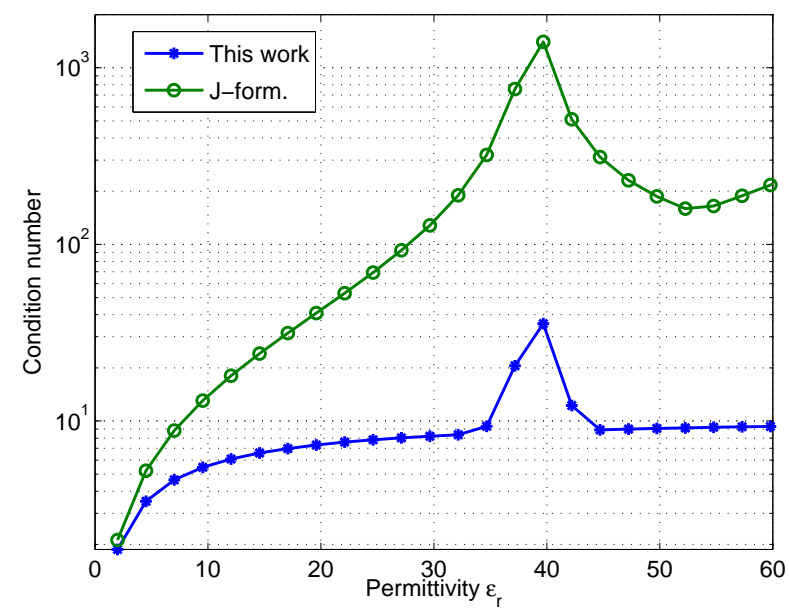

Fig. 6. Condition number as a function of permittivity. The size of the sphere is $k_{0} a=0.5$. The sphere has a physical resonance around $\epsilon_{r} \approx 40$.

Fig. 7 shows the radar cross section as a function of permittivity, and Fig. 8 presents the number of GMRES iteration required to solve the system. Both methods give almost the same solution, but less GMRES iterations are needed to solve the system when the preconditioner is applied.

Next, we consider an inhomogeneous case. The scatterer is a cube of size $k l=0.5$ located at the origin with permittivities $\epsilon_{1}(x<0)$ and $\epsilon_{2}(x>0)$. The cube is discretized with 2433 tetrahedral elements, and the incident field is an $x$ polarized planewave propagating along $z$-axis. Fig. 9 presents the number of iterations as a function of permittivity $\epsilon_{2}$ while 


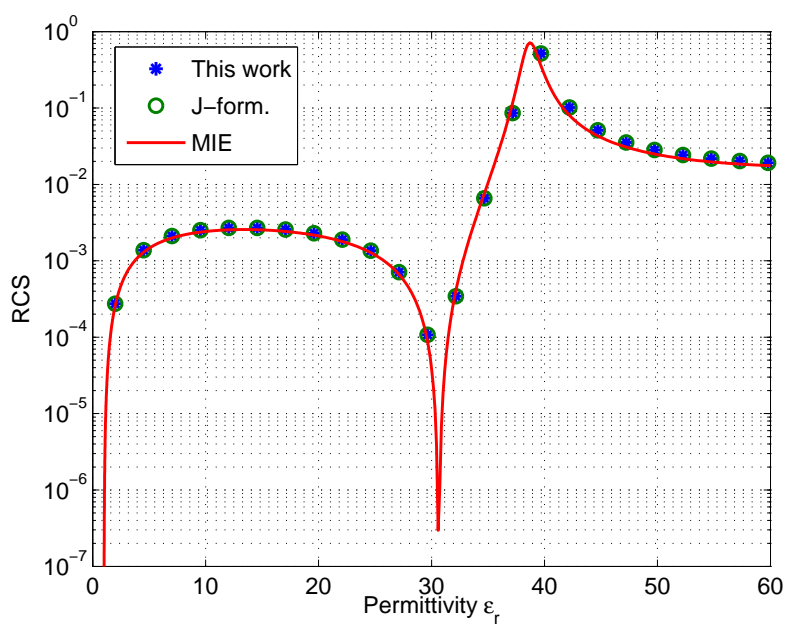

Fig. 7. Radar cross section of a dielectric sphere of size $k a=0.5$ as a function of permittivity.

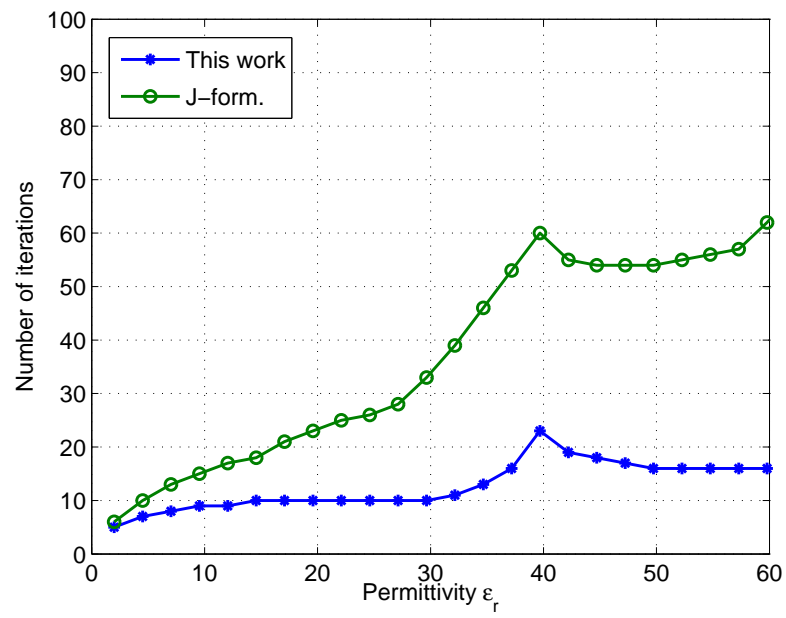

Fig. 8. Number of iterations required to solve the system as a function of permittivity. The GMRES solver without restart is applied with stopping criterion $10^{-5}$. The problem is the same as in Figs. 6 and 7 .

$\epsilon_{1}=10$. We do not observe any significant differences in the performance of the preconditioner in case of inhomogeneous cubes and homogeneous spheres as can be seen by comparing Figs. 8 and 9.

The discrete Helmholtz decompotition used in this paper does not suffer from the dense-discretization breakdown as opposed to the standard discrete decomposition. To demonstrate this, we consider an inhomogeneous lossy cube with $\epsilon_{1}=10+20 i$ and $\epsilon_{2}=40+10 i$. The edgelength of the cube is $l=1 \mathrm{~m}$, and the wavelength $\lambda_{0}=2 \pi \mathrm{m}$. The geometry is otherwise the same as in the previous example. Fig. 10 shows the number of iterations as a function of the number of unknows. Clearly, the convergence is independent of the mesh density as predicted. Also we can see that the preconditioner works for inhomogeneous as well as lossy dielectrics too.

Finally, we study a lossy dielectric cube. The size parameter of the cube is $k l=2$, and the real part of the permittivity $\epsilon_{r}=4$. The number of GMRES iterations is plotted as a

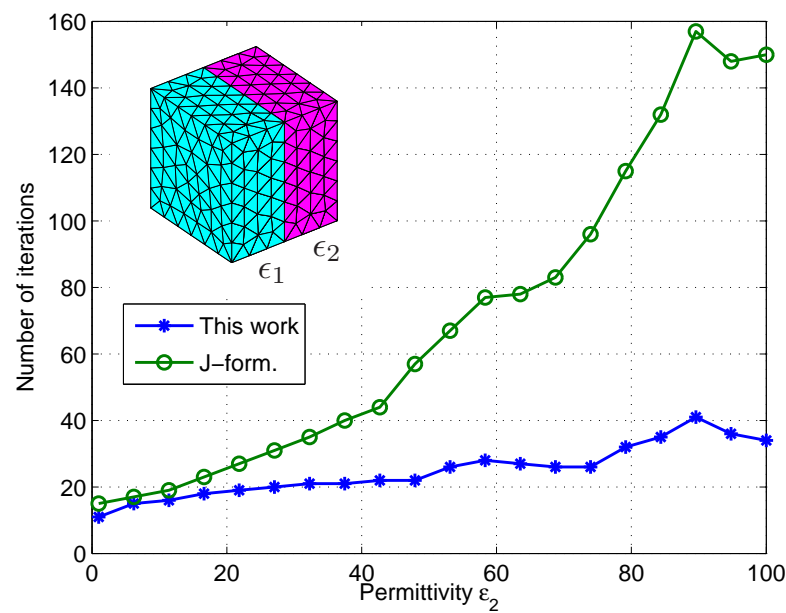

Fig. 9. Iteration count with respect to the permittivity $\epsilon_{2}(x>0)$. The scatterer is an inhomogeneous cube of size $k l=0.5$ located at the origin with $\epsilon_{1}=10(x<0)$.

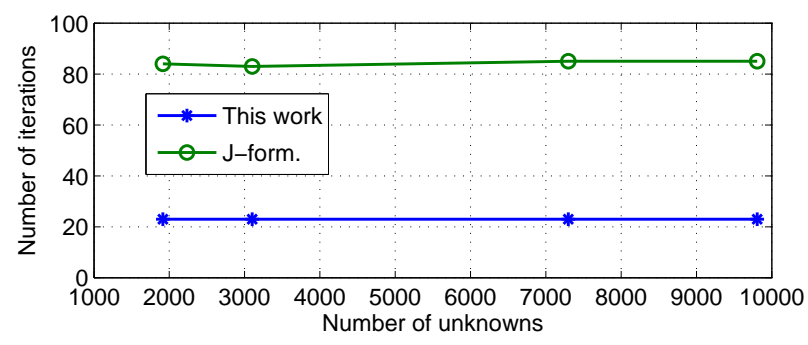

Fig. 10. Iteration count with respect to the number of unknowns. The scatterer is a lossy inhomogeneous cube of size $k l=1$ located at the origin with $\epsilon_{1}=10+20 i(x<0)$ and $\epsilon_{2}=40+10 i(x>0)$.

function of the imaginary part of the permittivity in Fig. 11. We can observe that also in the lossy case, the preconditioner decreases the iteration count.

\section{CONCLUSiOnS}

Spectral properties of the volume integral operator have been reviewed by performing an orthogonal decomposition in $L^{2}(\Omega)^{3}$. The $L^{2}(\Omega)^{3}$ vector space is decomposed into three orthogonal subspaces, solenoidal, irrotational, and gradients of harmonic subspaces. It has been shown that these orthogonal subspaces scale differently with respect to the permittivity, and it has a negative effect on the conditioning of the system. This explains why iterative solvers tend to converge very slowly when the contrast in the permittivity function is large.

We have proposed a preconditioner for the VIE that is based on the discrete Helmholtz decomposition. The discrete decomposition is implemented by applying projection operators that derive from the standard transformation matrix representation of solenoidal and irrotational basis functions. This technique leads to a well-posed system matrix whose condition number is independent on the mesh density and almost independent on the permittivity. The preconditioner is purely multiplicative, and therefore, relatively easy to implement for existing VIE solvers. In addition, this technique can be applied to other 


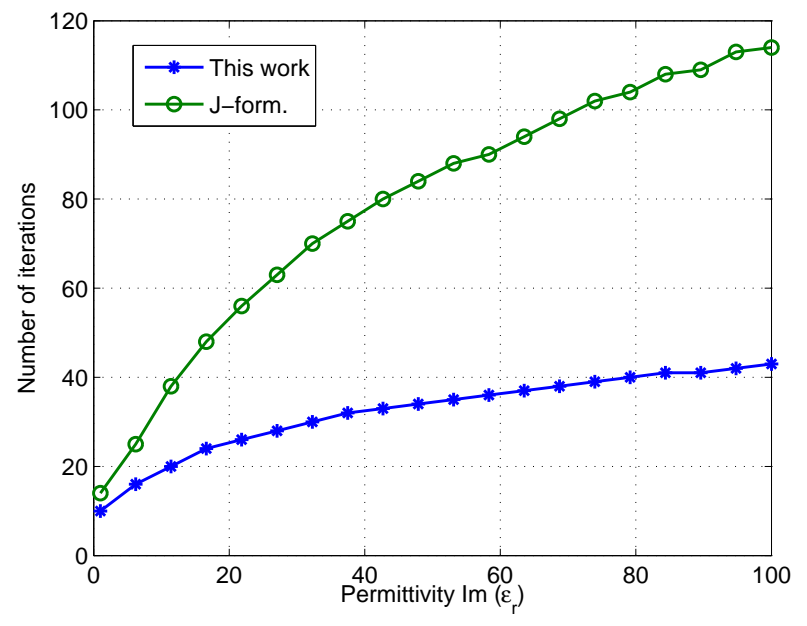

Fig. 11. Number of iterations required to solve the system as a function of the imaginary part of the permittivity. The scatterer is a cube of size $k l=2$ and the real part of the permittivity is 4 . The GMRES solver without restart is applied with stopping criterion $10^{-5}$.

VIE formulations as long as the proper discrete Helmholtz or quasi-Helmholtz decomposition is known.

Numerical examples show that the proposed preconditioner improves the conditioning of the system as well as decreases the number of iterations required to solve the problem. However, the pseudo-inverses required in construction of the projection operators cannot be computed in advance and stored. They must be computed iteratively in each iteration step, and preconditioners based on (auxiliary space) algebraic multigrids should be applied for efficient computations.

\section{REFERENCES}

[1] V. Rokhlin, "Rapid solution of integral equations of scattering theory in two dimensions", Journal of Computational Physics, vol. 86, no. 2, pp. 414-439, Feb. 1990.

[2] C.-C. Lu, "A fast algorithm based on volume integral equation for analysis of arbitrarily shaped dielectric radomes", IEEE Trans. on Antennas and Propagation, vol. 51, no. 3, pp. 606-612, Mar. 2003.

[3] E. Bleszynski, M. Bleszynski, and T. Jaroszewicz, "AIM: Adaptive integral method for solving large-scale electromagnetic scattering and radiation problems", Radio Science, vol. 31, no. 5, pp. 1225-1251, Sept. 1996.

[4] J.R. Phillips, and J.K. White, "A precorrected-FFT method for electrostatic analysis of complicated 3-D structures", IEEE Trans. ComputerAided Design of Integrated Circuits and Systems, vol. 16, no. 10, pp. 1059-1072, Oct. 1997.

[5] W. C. Chew, J.-M. Jin, E. Michielssen and J. Song, Fast and efficient algorithms in computational electromagnetics, Artech House, Boston, 2001.

[6] J. Markkanen, C-C. Lu, X. Cao, and P. Ylä-Oijala, "Analysis of volume integral equation formulations for scattering by high-contrast penetrable objects", IEEE Trans. Antennas and Propagation, vol. 60, no. 5, pp. 2367-2374, May 2012.

[7] M. Costabel, E. Darrigrand, and E.H. Koné, "Volume and surface integral equations for electromagnetic scattering by a dielectric body", Journal of Computational and Applied Mathematics, vol. 234, no. 6, pp. 1817-1825, Jul. 2010.

[8] G. C. Hsiao, "Boundary elements methods An overview", Applied Numerical Mathematics, vol. 56, pp. 1356-1369, 2006.

[9] G. C. Hsiao and R. E. Kleinman, "Mathematical foundations for error estimation in numerical solutions of integral equations in electromagnetics", IEEE Trans. Antennas Propagation, vol. 45, no. 3, pp. 316-328, Mar. 1997.
[10] M. Costabel, E. Darrigrand, and H. Sakly, "The essential spectrum of the volume integral operator in electromagnetic scattering by a homogeneous body", Comptes Rendus Mathematique, vol. 350, no. 3-4, pp. 193-197, Feb. 2012.

[11] M. C. van Beurden and S. J. L. van Eijndhoven, "Well-posedness of domain integral equations for a dielectric object in homogeneous background", J. Eng. Math(2008) 62:289-302.

[12] N. V. Budko and A. B. Samokhin, "Spectrum of the volume integral operator of electromagnetic scattering", SIAM J. Sci. Comput., vol. 28 , no. 2, pp. 682-700, 2006.

[13] J. Markkanen, P. Ylä-Oijala, and S. Järvenpää, "Volume integral equation methods in computational electromagnetics", International Conference on Electromagnetics in Advanced Applications (ICEAA), pp. 880-883, 9-13 Sept. 2013.

[14] M. C. van Beurden and S. J. L. van Eijndhoven, "Gaps in present discretization schemes for domain integral equations", International Conference on Electromagnetics in Advanced Applications, ICEAA 2007, Torino.

[15] H. Bhatia, G. Norgard, V. Pascucci, and P.-T. Bremer, "The HelmholtzHodge Decomposition - Survey", IEEE Trans. Visualization and Computer Graphics, vol. 19, no. 8, pp. 1386-1404, Aug. 2013.

[16] L. E. Sun and W. C. Chew, "A novel formulation of the volume integral equation for electromagnetic scattering", Waves in Random and Complex Media, vol. 19, no. 1, pp. 162-180, Feb. 2009.

[17] F. Andriulli, K. Cools, I. Bogaert, and E. Michielssen, "On a WellConditioned Electric Field Integral Operator for Multiply Connected Geometries", IEEE Trans. Antennas and Propagation, vol. 61, no. 4, pp. 2077-2087, Apr. 2013.

[18] F. Andriulli, K. Cools, I. Bogaert, and E. Michielssen, "Rapidly converging electromagnetic simulations in the entire frequency spectrum without the search for global loops", Proceedings of 2013 URSI International Symposium on Electromagnetic Theory (EMTS), pp. 45-48, May 2013.

[19] M.-K. Li and W. C. Chew, "Applying divergence-free condition in solving the volume integral equation", Progress in Electromagnetic Research, PIER 57, pp. 311-333, 2006.

[20] J.-C. Nédélec, "A new family of mixed finite elements in $\boldsymbol{R}^{3}$ ", Numer. Math., vol. 50, pp. 57-81, 1986.

[21] W.L Briggs, V.E. Henson, and S.F. McCormick, A Multigrid Tutorial (2nd Edition), SIAM: Philadelphia, PA, 2000.

[22] T.V. Kolev, and P.S. Vassilevski, Auxiliary Space AMG for H(curl) Problems, editors, U. Langer, M. Discacciati, D.E. Keyes, and O.B. Widlund, and W. Zulehner, Domain Decomposition Methods in Science and Engineering XVII, vol. 60, in series, Lecture Notes in Computational Science and Engineering, Springer Berlin Heidelberg, 2008.

[23] R. Hiptmair, and J. Xu, Nodal auxiliary space preconditioning in H(curl) and H(div) spaces, SIAM J. Numer. Anal., vol. 45, No. 6, pp. 2483-2509, 2007.

[24] J. Markkanen, P. Ylä-Oijala, and A. Sihvola, "Discretization of volume integral equation formulations for extremely anisotropic materials", IEEE Trans. Antennas and Propagation, vol. 60, no. 11, pp. 5195-5202, Nov. 2012.

Johannes Markkanen received the MSc in Electrical Engineering from Helsinki University of Technology, Espoo, Finland, in 2009, and the DSc (Tech) from Aalto University, Espoo, Finland, in 2013. Currently, he is working as a post-doctoral researcher with the Department of Physics, University of Helsinki, Finland. His research interests include integral-equation methods in computational electromagnetics, and modeling of complex electromagnetic media and surfaces. 